Danielle Pham-Dinh
Jean-Louis Nussbaum
Jean-Luc Popot
Odile Boespflug-Tanguy
Pierre Landrieu
André Dautigny

\section{Mutations du gène codant pour les protéolipides de la myéline (PLP et DM20) et dysmyélinisations liées au chromosome $X$}

Le gène $P L P$, situé sur le chromosome $\mathrm{X}$, code pour le protéolipide de la myéline, protéine majeure de la myéline dans le système nerveux central. Un épissage alternatif du transcrit de ce gène aboutit à deux messagers et à deux protéines, l'une ayant certainement un rôle structural prédominant (le PLP) et l'autre pouvant jouer un rôle fonctionnel dans la différenciation ou la survie des oligodendrocytes (la DM20). Il existe chez l'homme (maladie de Pelizaeus-Metzbacher), la souris (jimpy), le rat $(m d)$ et le chien (shaking pup) des maladies dysmyélinisantes homologues, récessives liées à l'X, dues à des mutations du gène $P L P$. Ces mutations modifient toutes des acides aminés conservés entre les espèces et communs au PLP et à la DM20, et s'accompagnent d'une mort accélérée des oligodendrocytes.

\section{ADRESSES}

D. Pham-Dinh : chargée de recherches à l'Inserm. A. Dautigny : directeur de recherches au Cnrs. Cnrs URA 1488, bâtiment B, 9, quai SaintBernard, 75252 Paris Cedex 05, France.

J.-L. Nussbaum : directeur de recherches au Cnrs. UPR $417 \mathrm{Cnrs}$, centre de neurochimie, Strasbourg, France. J.-L. Popot : sousdirecteur de laboratoire au Collège de France. URA 1187, Institut de biologie physicochimique, Paris, France. O. BoespflugTanguy: neuropédiatre. Université de Clermont-Ferrand, France. P. Landrieu chef $d u$ service de neuropédiatrie. Hôpital du es mutants dysmyéliniques constituent d'excellents modèles permettant d'élargir notre compréhension du processus normal de la myélinisation. La caractérisation des mutants de souris jimpy et shiverer au niveau moléculaire a, notamment, apporté de nombreux enseignements sur la structure, la topologie et la fonction des protéines majoritaires impliquées dans la myélinisation. Il s'agit des protéolipides spécifiques de la myéline (le PLP et son isof orme la DM20) et des protéines basiques (MBP) qui représentent 70 à $80 \%$ de l'ensemble des protéines constitu- tives de la myéline centrale. Le phénotype jimpy de la souris, comme d'autres dysmyélinisations transmises selon le mode récessif lié à l'X chez l'animal (rats md et shaking pup) et chez l'homme (maladie de PelizaeusMerzbacher), est associé à une mutation ponctuelle du gène PLP. L'implication de ces mutations dans la mort des oligodendrocytes, cellules responsables de la synthèse de myéline dans le système nerveux central, suggère que les isoformes codées par le gène PLP pourraient jouer des rôles différents dans le développement du système nerveux, d'abord au cours de la différenciation précoce des cellules 
Tableau I

PRINCIPALES PROTÉINES DE LA MYÉLINE CARACTÉRISÉES

\begin{tabular}{|c|c|c|}
\hline Protéines & $\begin{array}{l}\text { Myéline } \\
\text { centrale }\end{array}$ & $\begin{array}{c}\text { Myéline } \\
\text { périphérique }\end{array}$ \\
\hline Protéine zéro (Po) & - & $>50 \%$ \\
\hline $\begin{array}{l}\text { Protéolipides } \\
\text { PLP } \\
\text { DM20 } \\
\text { (2 isoformes) }\end{array}$ & $\begin{array}{r}50 \% \\
5 \%\end{array}$ & $2 \times \begin{array}{r}\text { traces } \\
\text { traces }\end{array}$ \\
\hline $\begin{array}{l}\text { Protéines basiques de la } \\
\text { myéline (MBP) } \\
\text { (4-7 isoformes selon l'espèce) }\end{array}$ & $30 \%$ & $5-15 \%$ \\
\hline $\begin{array}{l}\text { Glycoprotéines associées } \\
\text { à la myéline (MAG) } \\
\text { ( } 2 \text { isoformes) }\end{array}$ & $1 \%$ & $<1 \%$ \\
\hline $\begin{array}{l}\text { 2', } 3^{\prime} \text {-nucléotide cyclique } \\
3^{\prime} \text {-phosphohydrolase (CNPase) } \\
\text { (2 isoformes) }\end{array}$ & $4 \%$ & $<1 \%$ \\
\hline
\end{tabular}

gliales, puis dans la myélinisation proprement dite.

\section{La myéline}

Parmi les processus majeurs du développement du système nerveux, la formation de myéline ou myélinisation est un phénomène relativement tardif qui parachève des structures nerveuses fonctionnelles puisqu'elle intervient après la croissance axonale. Chez l'homme, la myélinisation débute pendant la vie fœtale, reste très active jusqu'à l'âge de 2 ans et se poursuit jusqu'à la puberté ; chez les rongeurs, elle commence après la naissance et dure environ trois semaines.

Au cours du processus de myélinisation, certains axones vont s'entourer d'une structure membranaire compacte, enroulée en spirale : la gaine de myéline. Cette gaine est formée par les prolongements cytoplasmiques élaborés par des cellules gliales spécialisées, les cellules de Schwann dans le système nerveux périphérique (SNP), les oligodendrocytes dans le système nerveux central (SNC) [1]. La myéline a une composition très particulière, en effet elle contient $\mathrm{m} / \mathrm{s} n^{\circ} 7$, vol. 8, seplembre 92 et enfin grâce à la caractérisation moléculaire de nombreux mutants dysmyéliniques (voir pour une revue $[3,4])$. Le protéolipide PLP et son isoforme la DM20 constituent à eux seuls $50 \%$ des protéines de la myéline centrale. Ces molécules sont particulièrement intéressantes à étudier du fait des questions encore sans réponse relatives à leur topologie précise dans la myéline, à leur rôle respectif au cours du développement du système nerveux et à la variété des effets engendrés par leurs mutations, en particulier au niveau cellulaire.

\section{Dysmyélinisations liées au chromosome $X$ et au gène PLP}

La localisation, en 1986, du gène PLP sur le chromosome X chez l'homme [5] a suggéré fortement que ce gène pourrait être impliqué dans certains troubles de la myélinisation du SNC. En effet, des dysmyélinisations du SNC, transmises selon le mode récessif lié au chromosome $\mathrm{X}$, ont été décrites depuis longtemps chez les mammifères. Parmi celles-ci, la maladie de Pelizaeus-Merzbacher chez l'homme [6, 7], et, chez l'animal, les mutations des souris jimpy et jimpy ${ }^{m s d}$, du rat déficient en myéline $m d$ (pour une revue voir [8]) et du chien shaking pup ont fait l'objet d'études approfondies, tant du point de vue morphologique que biochimique et cellulaire. Ces mutations partagent entre elles un certain nombre de caractéristiques neuropathologiques évoquant un processus de dysmyélinisation : absence ou rares îlots de myéline dans la substance blanche du SNC, absence d'inflammation et d'atteinte des neurones, important déficit en oligodendrocytes mûrs, anomalies phénotypiques des astrocytes (autre type de cellules gliales du SNC).

Mutation jimpy (voir $m / s \quad n \circ 9$, vol. 2, p. 256)

Les critères génétiques et morphologiques décrits plus haut rendaient plausible la recherche d'une anomalie structurale au niveau du gène PLP. L'application des techniques de biologie moléculaire, et notamment de la cartographie à la nucléase S1, 
Tableau II

MUTATIONS DÉLÉTĖRES DU GĖNE PLP

\section{RÉFÉRENCES}

1. Gumpel M, Tourbah A, Baron-Van Evercoren A. La réparation myćlinique dans le SNC des mammifòres. médecine/sciences $1991 ; 7: 782-9$.

2. Lees MB, Brostoff SW. Proteins of mye lin. In : Morell P, ed. Myelin. New York Plenum Press, 1984 : 197-224.

3. Campagnoni AT, Macklin WB. Cellular and molecular aspects of the myclin protein gene expression. Mol Neurobiol 1988 ; 2 : $41-89$.

4. Popot JL, Pham-Dinh D, Dautigny A. Major myelin protcolipid : the 4- $\alpha$-helix topology. J Membrane Biol 1991; 120 233-46.

5. Mattei MG, Alliel PM, Dautigny A, et al. The gene encoding for the major brain protcolipid (PLP) maps on the q-22 band of the human X chromosome. Hum Genet $1986 ; 72: 352-3$

6. Pclizaeus F. Ucber eine cigentümliche Form spastischer Lähmung mit Cerebralerscheinungen auf hereditärer Grundlage (multiple Sklerose). Arch Psychiatr 1985; 16 : 698-710.

7. Merzbacher L. Eine eigenartige familiäre Erkrankungsform (Aplasia axialis extracorticolis congenita). Z Ges Neurol Psychiatr $1910 ; 3$ : 1-138.

8. Hogan EL, Greenfield S. Animal models of genetic disorders of myelin. In : Morel P, ed. Myelin. New York: Plenum Press, 1984: 489-534.

9. Dautigny A, Mattei MG, Morello D, et al. The structural gene coding for myelinassociated protcolipid protein is mutated in jimpy mice. Nature 1986 ; 321 : 867-75.

10. Morello D, Dautigny A, Pham-Dinh D, Jollès P. Myclin proteolipid (PLP and DM20) transcripts are deleted in jimpy mutant mice. EMBO J 1986 ; 5 : 3489-93.

11. Nave KA, Bloom FE, Milner RJ. A single nucleotide difference in the gene for myelin proteolipid protein defines the jimpy mutation in mouse. $J$ Neurochem $1987 ; 49$. 1873-7.

12. Roussel G, Neskovic NM, Trifilieff E Artault JC, Nussbaum JL. Arrest of proteolipid transport through the Golgi apparatus in jimpy brain. $J$ Neurocytol $1987 ; 16$ 195-204

13. Skoff RP, Knapp PE. Jimpy phenotype expression in relation to proteolipid protein appearance. In : Duncan ID, Skoff RP, Colman D, eds. Myelination and Dysmyelination. New York: Annals of the New York Academy of Sciences, $1990 ; 122-33$

\begin{tabular}{|c|c|c|c|}
\hline \multicolumn{4}{|c|}{$\begin{array}{c}\text { Tableau ॥ } \\
\text { MUTATIONS DÉLÉTĖRES DU GĖNE PLP }\end{array}$} \\
\hline Mutant & Mutation & Exon & Références \\
\hline Souris & & & \\
\hline jimpy & $\begin{array}{c}\text { Ala } \rightarrow \text { Glu } \\
\text { énissae }\end{array}$ & intron IV & 9, 10 \\
\hline jimpymsd & $\mathrm{Ala}^{242} \rightarrow \mathrm{Val}$ & VI & 24 \\
\hline Rat & & & \\
\hline md & $\mathrm{Thr}^{74} \rightarrow$ Pro & III & 25 \\
\hline $\begin{array}{l}\text { Chien } \\
\text { shaking pup } \\
\text { Homme }\end{array}$ & $\mathrm{His}^{36} \rightarrow$ Pro & II & 26 \\
\hline P-M 1 & $\operatorname{Trp} 162 \rightarrow \operatorname{Arg}$ & IV & 17 \\
\hline P-M 2 & $\mathrm{Pro}^{215} \rightarrow \mathrm{Ser}$ & v & 18 \\
\hline P-M 3 & Pro $^{14} \rightarrow$ Leu & II & 19 \\
\hline P-M 4 & Thr $155 \rightarrow \|$ e & IV & 20 \\
\hline P-M 5 & $\mathrm{Val}^{218} \rightarrow \mathrm{Phe}$ & $\mathrm{v}$ & 21 \\
\hline P-M 6 & $\begin{array}{l}\text { délétion totale } \\
\text { du qène }\end{array}$ & I-VII & 22 \\
\hline P-M 7 & $\mathrm{Tyr}^{206} \rightarrow \mathrm{Cys}$ & IV & 23 \\
\hline
\end{tabular}

a permis de démontrer que les transcrits du gène PLP étaient partiellement délétés chez ce mutant [9]. Cette délétion était retrouvée dans la même région de la séquence des deux transcrits PLP et DM20, suggérant ainsi que ces deux isoformes sont le produit de l'épissage alternatif d'un même transcrit primaire du gène PLP [10]. La délétion jimpy n'ayant pas été retrouvée au nivcau génomique, on pouvait supposer qu'elle pourrait être due à un épissage défectueux. Cette hypothèse a pu être, par la suite, vérifiée au niveau génomique par la démonstration que lc gène $P L P$, chez jimpy, comportait une transition $\mathrm{A} \rightarrow \mathrm{G}$ au niveau de la séquence consensus d'un site accepteur d'épissage, ce qui aboutit à l'élimination d'un exon et à un changement du cadre de lecturc [11].

Ces résultats de génétique moléculaire ont été confirmés, au nivcau cellulaire, par l'emploi de méthodes immunohistochimiques à l'échclle de la microscopie électronique. Le protéolipide PLP de la souris jimpy, présentant une extrémité carboxyterminale modifiée, n'est pas reconnu par un anticorps anti-hexapeptide dirigé contre la séquence C-tcrminale de la protéine normale. La présence d'une protéine PLP mutée, bloquée sur lc lieu de sa synthèse dans le réticulum endoplasmique, est en revanche reconnuc, dans les rares oligodendrocytes survivants, par des anticorps dirigés contre la séquence entière dc la protéine normale [12]. La mutation jimpy est, par ailleurs, associée à une accélération de la différenciation des précurseurs des oligodendrocytes et à unc importante mort des oligodendrocytes mûrs (jusqu'à $24 \%$ de mort ccllulaire) dès le début de la myélinisation [13]. Cette mort cellulairc pathologique, dont les mécanismes ne sont pas encore élucidés, est bien le trait le plus étonnant de ce phénotype.

La maladie de PelizaeusMerzbacher (voir $m / s n^{\circ} 1$, vol. 6 , p. 70)

Les maladies regroupécs dans la littérature médicale sous le terme éponyme de Pelizacus-Merzbacher appartiennent au groupc des leucodystrophies soudanophiles. Le terme générique de "leucodystrophies" recouvre, pour le clinicien et le neuropathologiste, une grande variété de maladies, en règle générale génétiques, où les troubles de la myélinisation et/ou de la stabilité de la myéline constituent un phénomène prépondérant. Parmi ces leucodystro- 
phies, Pelizaeus (1885) [6] ct Merzbacher (1910) [7] individualisèrent, à partir d'une famille comportant 14 sujets atteints sur quatre générations, une affection d'évolution très lente caractérisée par : (1) son mode de transmission liée au chromosome X ; (2) sa présentation clinique homogène s'exprimant dès les pre- miers mois de la vie par une hypotonie associée à un nystagmus puis à un tremblement de la tête, des mouvements involontaires choréoathétosiques et plus tardivement des signes pyramidaux; (3) son aspect neuropathologique limité au SNC, sans atteinte axonale, avec persistance d'îlots de myéline périvasculaires rela- tivement conservés, situés sur le trajet des grands faisceaux myélinisés, donnant un aspect "tigroïde " de la substance blanche. Les études histochimiques ultéricures permirent de qualifier cette leucodystrophie de soudanophile du fait de la présence, dans la substance blanche du SNC, de graisses neutres se colorant par le

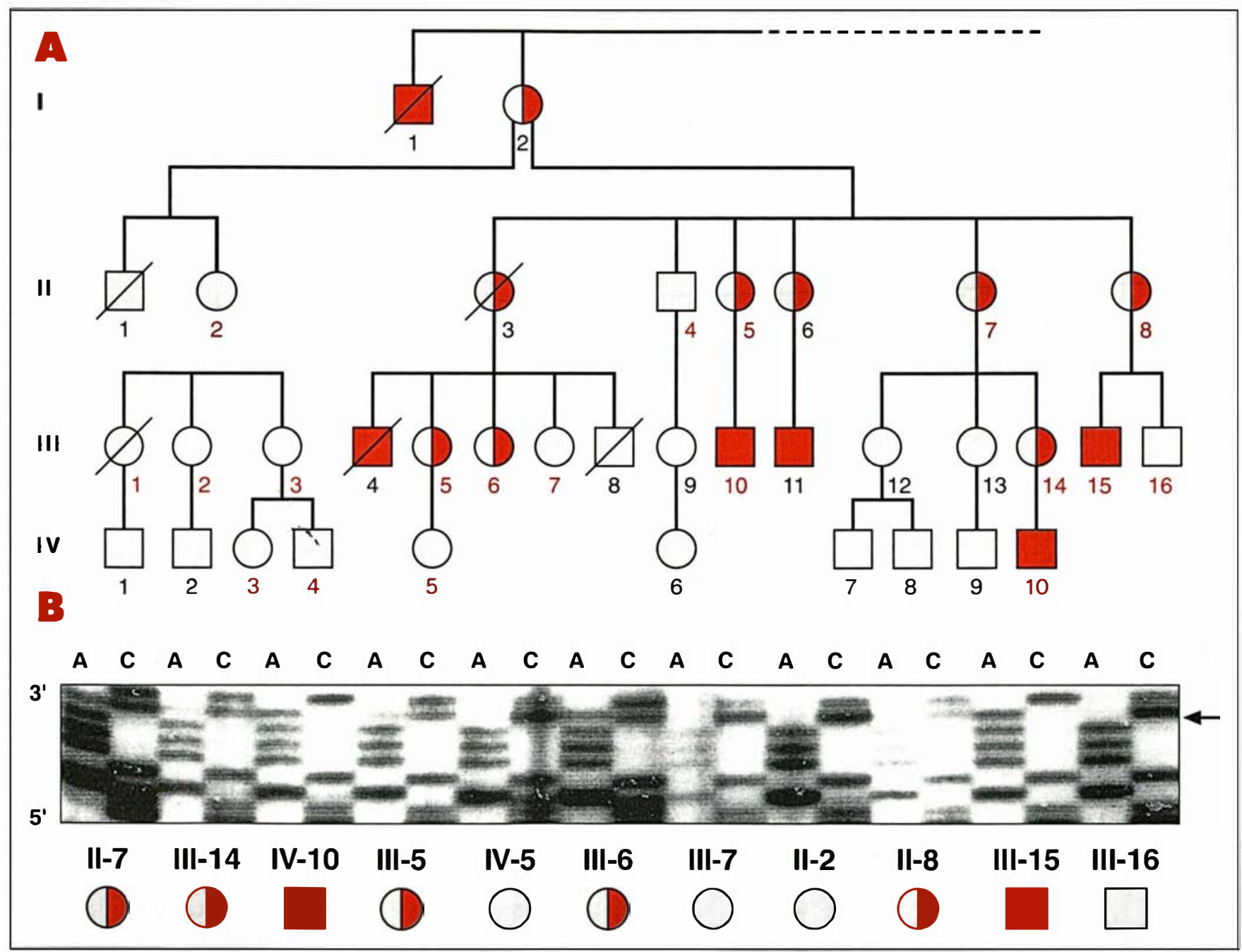

Figure 1. A. Pedigree partiel de la famille affectée par la mutation Val218 $\rightarrow$ Phe. Les générations sont indiquées par des chiffres romains. Les chiffres arabes rouges indiquent les individus dont le statut génomique a été déterminé. Les carrés rouges représentent les mâles atteints; les carrés gris, les mâles non atteints; les cercles moitié gris-moitié rouges représentent les femmes obligatoirement conductrices; les cercles gris représentent les femmes homozygotes saines ou celles dont le statut génomique n'a pas été déterminé ; une barre transversale représente un individu décédé. B. Séquençage des nucléotides $A$ et $C$ chez 11 sujets représentatifs de la famille dont le pedigree est représenté figure 1A. L'allèle normal et l'allèle muté sont identifiés par le séquencage du brin anti-sens de l'exon $V$ du gène PLP de sujets affectés (Phe: AAA), homozygotes sains (Val : CAA), et de femmes hétérozygotes transmettrices (Val/Phe : CAA/AAA). 


\section{RÉFÉRENCES}

14. Seitelberger F. Pelizacus-Merzbacher discasc. In : Vinken PJ, Bruyn GW, eds. Handbook of Clinical Neurology. Amsterdam : North Holland, $1970 ; 10: 150-202$.

15. Boulloche J, Aicardi J. PclizacusMerzbacher discase : clinical and nosological study. J Child Neurol 1986; 1 : 233-9.

16. Caro PA, Marks HG. Magnetic resonnance imaging and computed tomography in Pclizacus-Merzbacher discasc. Magn Res Imag $1990 ; 8$ : 791-6.

17. Hudson LD, Puckett C, Berndt J, Chan J, Gencic S. Mutation of the proteolipid protein gene PLP in a human X chromosome-linked disorder. Proc Natl Acad Sci USA 1989 ; 86 : 8128-31.

18. Gencic S, Abuclo D, Ambler M, Hudson LD. Pclizacus-Merzbacher discase : an $\mathrm{X}$-linked neurologic disorder of myclin metabolism with a novel mutation in the genc encoding protcolipid protein. $A m \mathrm{~J}$ Hum Genet 1989: 45 : 435-42.

19. Trofatter JA, Dlouhy SR, DeMcycr W, Conncally PM, Hodes ME. PclizacusMerzbacher discase : tight linkage to proteolipid protein gene exon variant. Proc Nat Acad Sci USA 1989; 86 : 9427-30.

20. Pratt VM, Trofatter JA, Schinzcl A Dlouhy SR, Conncally PM, Hodes ME. A new mutation in the proteolipid protein (PLP) genc in a German family with Pelizacus-Merzbacher discasc. $A m \mathrm{~J}$ Med Genet 1991 ; 38 : 136-9.

21. Pham-Dinh D, Popot JL, Bocspflug Tanguy $\mathrm{O}$, et al. Pclizacus-Merzbacher discase : a valine to phenylalanine point mutation in a putative extracellular loop of myclin protcolipid. Proc Natl Acad Sci USA $1991 ; 88: 7562-6$.

22. Raskind HW, Williams CA, Hudson LD, Bird TD. Complete deletion of the protcolipid protein gene (PLP) in a family with $\mathrm{X}$-linked Pelizacus-Mcrzbacher discasc. $A m$ J Hum Genet 1991; 49 : 1355-60.

23. Bridge PJ, D'Souza CR, van Oost BA

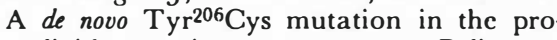
tcolipid protein gene causes Pelizacus Merzbacher discasc. Am J Hum Genet 1991 ; $49: 183$ (5)

24. Gencic S, Hudson LD. Conservative amino-acid substitution in the myclin protcolipid protcin of jimpy msd micc. J Neurosci $1990 ; 10: 117-24$

25. Boison D, Stoffel W. Myclin-deficient rat : a point mutation in exon III $(\mathrm{A} \rightarrow \mathrm{C}$, $\mathrm{Thr}^{75} \rightarrow$ Pro) of the myclin protcolipid protein causes dysmyclination and oligoden- noir Soudan, démontrant ainsi que le catabolisme de la myéline était normal dans cette affection. Cette caractéristique s'opposait ainsi aux autres leucodystrophies où était mise en évidence, dans la substance blanche, l'accumulation de produits anormaux (ex. : leucodystrophies métachromatiques). Par extension abusive, le terme de Pelizaeus-Merzbacher fut appliqué, dans la littérature neurologique, aux patients ayant des caractéristiques neuropathologiques proches. Seitelberger (1970) [14] définit ainsi sept formes selon l'âge de début et l'évolution. Plus récemment, Boulloche et Aicardi [15], à propos de sept familles, ont redéfini le cadre nosologique de cette affection, réservant le terme de PelizaeusMerzbacher aux formes liées à l'X, également appelées dysmyélinisations liées à l'X, pour éviter toute confusion. Les critères diagnostiques, cliniques et génétiques, qu'ils ont ainsi rappelés prennent actuellement toute leur importance, car le trouble de formation de la myéline limité au SNC peut être objectivé par des explorations neurophysiologiques (potentiels évoqués, vitesse de conduction nerveuse) et neuroradiologiques (imagerie cérébrale en résonance magnétique nucléaire [16]), permettant de porter un diagnostic précoce.

La maladie de Pelizaeus-Merzbacher ainsi définie a des caractéristiques phénotypiques et génétiques proches de celles du mutant jimpy et des autres modèles animaux de dysmyélinisation liée au sexe. Les résultats obtenus chez la souris jimpy ont conduit différentes équipes à rechercher une implication du gène $P L P$ dans la genèse de la maladie de PelizaeusMerzbacher. Différentes substitutions d'acide aminé, ainsi qu'une délétion totale du gène, ont effectivement été trouvées associées à cette affection ([17-23] (Tableau II, p. 666)).

Nous avons étudié les régions exoniques codantes du gène PLP de patients présentant le phénotype Pelizaeus-Merzbacher et appartenant à 15 familles non apparentées, à partir de banques préparées avec de l'ADN génomique pathologique, ou par la technique d'amplification à la polymérase (PCR).

Dans une seule famille, nous avons pu mettre en évidence, chez les trois garçons atteints analysés, une mutation ponctuelle $\mathrm{G} \rightarrow \mathrm{T}$ dans l'exon $\mathrm{V}$, produisant ainsi un remplacement de la valine 218 par une phénylalanine. La recherche de cette mutation chez 21 membres de cette famille répartis sur cinq générations a permis de démontrer une stricte coségrégation entre la transmission de la maladie et l'allèle muté (figures $1 \mathrm{~A}$ et $1 B$, p. 667) [21].

Dans une autre famille, une biopsie de nerf périphérique de l'un des patients a permis d'amplifier par PCR puis de séquencer les transcrits PLP et DM20, qui sont minoritaires dans ce tissu. Nous avons ainsi pu montrer que ces transcrits présentaient un épissage correct et une séquence codante normale (à l'exception d'une mutation silencieuse) [21]. Ce cas est à rapprocher de celui des autres malades étudiés au laboratoire ou dans d'autres équipes $[18,20,21]$ pour lesquels aucune anomalie n'a pu être trouvée dans les régions exoniques codantes du PLP. On peut émettre l'hypothèse que, si le gène PLP était impliqué dans tous ces cas, les régions régulatrices du gène pourraient être altérées.

Pour répondre à cette question, nous avons entrepris des études de liaison génétique à partir de 18 familles informatives. La mise en évidence d'une liaison étroite entre la maladie, la sonde ADNc PLP et des marqueurs polymorphes proches [27] suggèrent une homogénéité quant au gène en cause dans cette affection, du moins si l'on s'en tient aux critères diagnostiques stricts que nous avons utilisés [15] pour la sélection de nos familles. Cette homogénéité génétique permet actuellement, dans les familles de patients atteints de cette affection où aucune anomalie dans les régions exoniques codantes du gène PLP n'a pu être mis en évidence, de déterminer les femmes transmettrices et de faire un diagnostic anténatal grâce à l'utilisation des marqueurs polymorphes (dans et proche du gène $P L P$ ) démontrés liés à la maladie, en attendant la mise en évidence du défaut moléculaire au niveau des régions régulatrices.

Au cours de cette étude, nous avons aussi noté, dans le SNP, une régulation différente de l'épissage alternatif des deux isoformes du PLP par 
rapport à celle observée, dans le SNC. En effet, contrairement au fait bien établi que le PLP constitue l'isoforme majoritaire de la myéline centrale (50\% du contenu protéique), on observe dans le nerf périphérique, une prépondérance des transcrits DM20 par rapport au PLP [21] (indépendamment du fait que la quantité totale des deux isoformes est extrêmement faible dans ce tissu ; voir Tableau I). Cette caractéristique a été retrouvée chez le rat, aussi bien au niveau des transcrits, après amplification des $\mathrm{ADNc}$, que par analyse des protéolipides par immunoempreinte (figure 2) [28].

\section{Conservation de la séquence du PLP parmi les espèces}

La comparaison des séquences protéiques déduites de l'ADNc ou du gène codant pour le PLP chez l'homme, le rat, la souris et le chien, fait apparaître un degré de conservation étonnant (un seul acide aminé différent chez le chien) [26]. Ce fait pourrait refléter l'existence de nombreux sites fonctionnels le long de cette molécule, ce qui expliquerait la très grande vulnérabilité des fonctions du PLP et de la DM20 à toute substitution, même conservative (comme la mutation Ala $\rightarrow$ Val chez la souris jimpy ${ }^{m s}$, par exemple). L'ADNc du PLP de poulet, caractérisé récemment, présente en revanche un certain nombre de substitutions neutres (18 différences) par rapport à celui des mammifères [29]. Cependant, il est tout à fait remarquable de constater que les mutations délétères répertoriées à ce jour chez les mammifères (Tableau II) ne touchent que des acides aminés totalement conservés parmi les différentes espèces étudiées.

\section{Topologie transmembranaire des protéolipides PLP et DM20}

Pour tenter de comprendre l'impact possible d'une mutation sur la structure et la fonction du PLP, il est indispensable de disposer d'un modèle structural de la protéine. On ne sait pas actuellement déduire directement de la séquence des aci$\mathrm{m} / \mathrm{s} n^{\circ} 7$, vol. 8 , seplembre 92

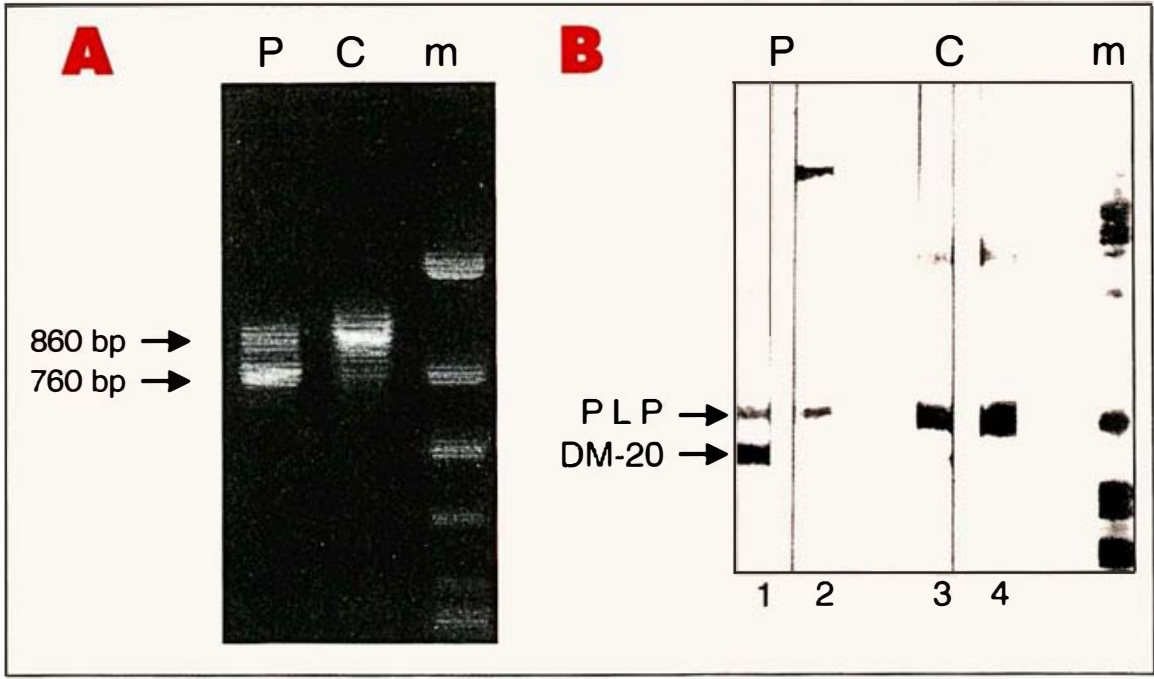

Figure 2. A. Séparation, sur gel d'agarose à 1,5\%, des produits d'amplification obtenus à partir des ADNc de PLP et de DM20. Les ADNc ont été synthétisés à partir d'ARN total du nerf périphérique $(P)$, ou du système nerveux central (C) de rat de 14 jours. $m$ : marqueurs de poids moléculaire. B. Immunoempreinte réalisée sur un extrait protéique de nerf périphérique (P) et de myéline du système nerveux central (C) de rat adulte. L'antihexapeptide C-terminal du PLP détecte le PLP et la DM2O (pistes 1 et 3) ; I'antitridécapeptide 117-129 (pistes 2 et 4), correspondant à la délétion DM20, détecte uniquement le PLP. $m$ : SDS-PAGE des protéines de la myéline du système nerveux central $(100 \mu \mathrm{g})$ après coloration au bleu de Coomassie.

des aminés d'une protéine, sa structure tridimensionnelle détaillée. Toutefois, dans le cas des protéines transmembranaires intégrales comme le PLP, les contraintes de l'environnement membranaire font que la région transmembranaire de la protéine est le plus souvent composée d'un assemblage d'hélices $\alpha[30,31]$. Ces hélices comportent 20-30 acides aminés majoritairement hydrophobes et peuvent donc se repérer dans la séquence. La figure $3 A$ p. 671, montre la variation de l'hydrophobicité locale le long de la séquence du PLP, et la position de quatre segments très hydrophobes, que nous présumons former chacun une hélice transmembranaire (numérotées de A à D). Le degré de fiabilité de ce genre d'analyse est variable. Dans le cas du PLP, l'hydrophobicité est très élevée, et la probabilité que ces segments ne soient pas transmembranaires est très faible.

Afin de déterminer l'orientation du modèle dans la membrane, on peut se fonder sur la distribution des résidus basiques dans les segments extramembranaires. Ces résidus, en effet, jouent un rôle important lors de la biosynthèse et, en substance, contrôlent la polarité de l'insertion. L'examen de leur distribution nous amène à penser que les extrémités N- et Cterminales du PLP se trouvent dans le cytosol (figure 3B). A nouveau, cette orientation est compatible avec les données expérimentales, mais non prouvée.

Il n'existe pas actuellement de méthode générale permettant de prédire comment les quatre hélices s'associent pour former un faisceau. Toutefois, il est souvent possible de deviner, pour une hélice donnée, quelle face elle tourne vers l'intérieur du faisceau et quelle face elle expose aux lipides. Cette dernière est généralement plus hydrophobe, et elle varie plus vite au cours de l'évolution. Cette asymétrie est illustrée par la figure $3 C$ pour l'hélice $C$, vue en projection sur le plan de la membrane. La face la plus hydrophobe est ombrée. Nous avions proposé, lors de la publication de notre modèle, qu'elle faisait face aux lipides. Depuis lors, des éléments de la séquence des PLP de poulet et de xénope ont été 


\section{RÉFÉRENCES}

26. Nadon NL, Duncan ID, Hudson LD A point mutation in the proteolipid gene of the shaking pup interrupts oligodendrocyte devclopment. Development $1990 ; 110$ : 529-37.

27. Boespflug O, Cavagna A, Pham-Dinh D, Melki J, Dautigny A, Dastugue B. Linkage analysis in human X-linked dysmyelinating diseases (Pelizaeur-Merzbacher disease). Am J Hum Genet 1991; 49: 183 (5).

28. Pham-Dinh D, Birling MC, Roussel G Dautigny A, Nussbaum JL. Proteolipid DM20 predominates over PLP in periphe ral nervous system. Neuro Report 1991; 2 : 89-92.

29. Schliess F, Stoffel W. Evolution of the myclin integral membrane proteins of the central nervous system. Biol Chem HoppeSeyler $1991 ; 372$ : 865-74.

30. Popot JI, Engelman DM. Membrane protein folding and oligomerization : the two stage model. Biochemistry $1990 ; 29$ : 4031-7.

31. Popot JL, de Vitry C, Atteia A. Folding and assembly of integral membrane proteins. An introduction. In : White $\mathrm{SH}$ cd. Membrane Protein Structure: Experimental Approaches. Oxford : Oxford University Press, 1992 (sous presse).

32. Schindler P, Luu B, Sorokine O, Trifilieff $\mathrm{E}, \mathrm{V}$ an Dorsselaer A. Devclopmenta study of proteolipids in bovine brain : a novel proteolipid and DM20 appear before proteolipid protein during myelination. $J$ Neurochem 1991 ; 55 : 2079-85.

33. Ikenaka K, Kagawa T, Mikoshiba K Selective expression of DM20, an alternatively spliced myelin proteolipid protein gene product, in devcloping nervous system and in non-glial cells. J Neurochem 1992 (sous presse).

34. Timsit SG, Bally-Cuif L, Colman DR Zalc B. DM20 mRNA is expressed during the embryonic development of the nervous system of mouse. $J$ Neurochem 1992 (sous presse).

35. Dutly F, Schwab ME. Neurons and astrocytes influence the development of purified O-2A progenitor cells. Glia 1991; 4 559-71.

36. Lachapelle F, Lapie P, Campagnoni AT, Gumpel M. Oligodendrocytes of the jimpy phenotypc can be partially restored by environmental factors in vivo. I Neurosci Res $1991 ; 29: 235-43$. établis, qui couvrent cette région [29]. Les résidus marqués sur fond blanc diffèrent entre le poulet ou le xénope et l'homme, les résidus sur fond noir sont conservés. Les résidus variables tendent effectivement à se regrouper sur la face censée faire face aux lipides.

Ce type de modélisation permet d'envisager le rôle fonctionnel des différentes régions de la séquence et de formuler des hypothèses concernant le mode d'action des mutations. Ainsi la substitution $\mathrm{Val}^{218} \rightarrow$ Phe que nous avons découverte chez des patients souffrant du syndrome de PelizaeusMerzbacher affecte un résidu que notre modèle place dans l'espace extracellulairc [21] (figure 3B). Parm les différentes hypothèses possibles, on attachera une attention particulière à celles qui postulent une interaction défectueuse avec des molécules situées dans le lumen du réticulum endoplasmique, lors de la biosynthèse, ou dans le milicu extracellulaire. Une autre mutation responsable d'un syndrome dc Pelizaeus-Merzbacher, récemment découverte par Pratt et al. (1991) [20], affecte le rćsidu $\operatorname{Thr}{ }^{155}$ que notre modèle place sur la face de l'hélice C tournée vers l'intéricur du faisceau d'hélices (figure 3C). On pcut penser qu'elle déstabilise le PLP, et/ou affecte sa structure tridimensionnelle, en empêchant les hélices de

s'associer correctement les unes avec les autres.

Un mot de prudence au sujet de ces prédictions : un modèle est fait pour guider l'expérience, non pour se substituer à elle. Même si le modèle décrit dans cet article est compatible avec un grand nombre d'observations, dont certaines effectuées postérieurement à sa publication, la topologie proposée ne doit pas être considérée comme établie tant qu'elle n'a pas été validée expérimentalement.

\section{Un gène $\rightarrow$ deux protéolipides $\rightarrow$ deux fonctions différentes?}

Il y a quelques années encore, le PLP et la DM20 étaient considérés comme ayant un rôle essenticllement structural. Cela reste toujours vrai en ce qui concerne le PLP. En effet, en tant que protéinc transmembranaire majoritaire de la myéline du SNC, le PLP est vraisemblablement impliqué dans l'adhérence des tours successifs de la membranc des cellules myélinisantes au niveau de sa face extracytoplasmique et, probablement, dans la compaction des faces internes. Une preuve directe de ce rôle architectural a été apportée par le fait que, chez certains mutants comme le rat $m d$, la périodicité de la myćline passe de 11 à $9 \mathrm{~nm}$, en absence du PLP.

Figure 3. A. Profil d'hydrophobicité du PLP. Les propriétés locales d'hydrophobicité du PLP le long de sa séquence sont analysées en calculant point par point l'hydrophobicité moyenne d'un segment de 17 acides aminés. Les quatre régions susceptibles de former des hélices $\alpha$ transmembranaires hydrophobes forment autant de pics d'hydrophobicité élevée, notés A à D. (Voir [4].) B. Position des mutations délétères dans le modèle topologique du PLP. En rouge, les substitutions causant le syndrome de Pelizaeus-Merzbacher (P-M) ou des maladies génétiques animales apparentées. Voir Tableau II et [21]. La région de la séquence délétée dans la DM20 est représentée par une ligne pointillée. Les deux régions encadrées présentent des similitudes de séquence avec une région cytosolique des récepteurs couplés aux protéines $G$ (Rho: rhodopsine ; $\beta_{1} A R$ : récepteur $\beta_{1}$-adrénergique). Dans le PLP et la rhodopsine, les résidus cystéyl représentés en gras (C) sont palmitoylés. C. Vue schématique de la troisième hélice $\alpha$ transmembranaire du PLP (hélice C), en projection sur le plan de la membrane. Sur la base de la distribution des résidus hydrophiles et hydrophobes, la face grisée est présumée faire face aux lipides, la face claire être tournée vers l'intérieur du faisceau d'hélices $\alpha$. Cette hypothèse est en accord avec la répartition des variations neutres (indiquées sur fond blancl entre l'homme, le xénope et le poulet : la face exposée aux lipides varie plus rapidement au cours de l'évolution. Les résidus indiqués en blanc sur fond noir ou rouge sont invariants dans toutes les séquences disponibles du PLP normal. Les deux substitutions indiquées par un fond rouge sont liées à un syndrome de Pelizaeus-Merzbacher (Tableau II). (Voir [4].) 


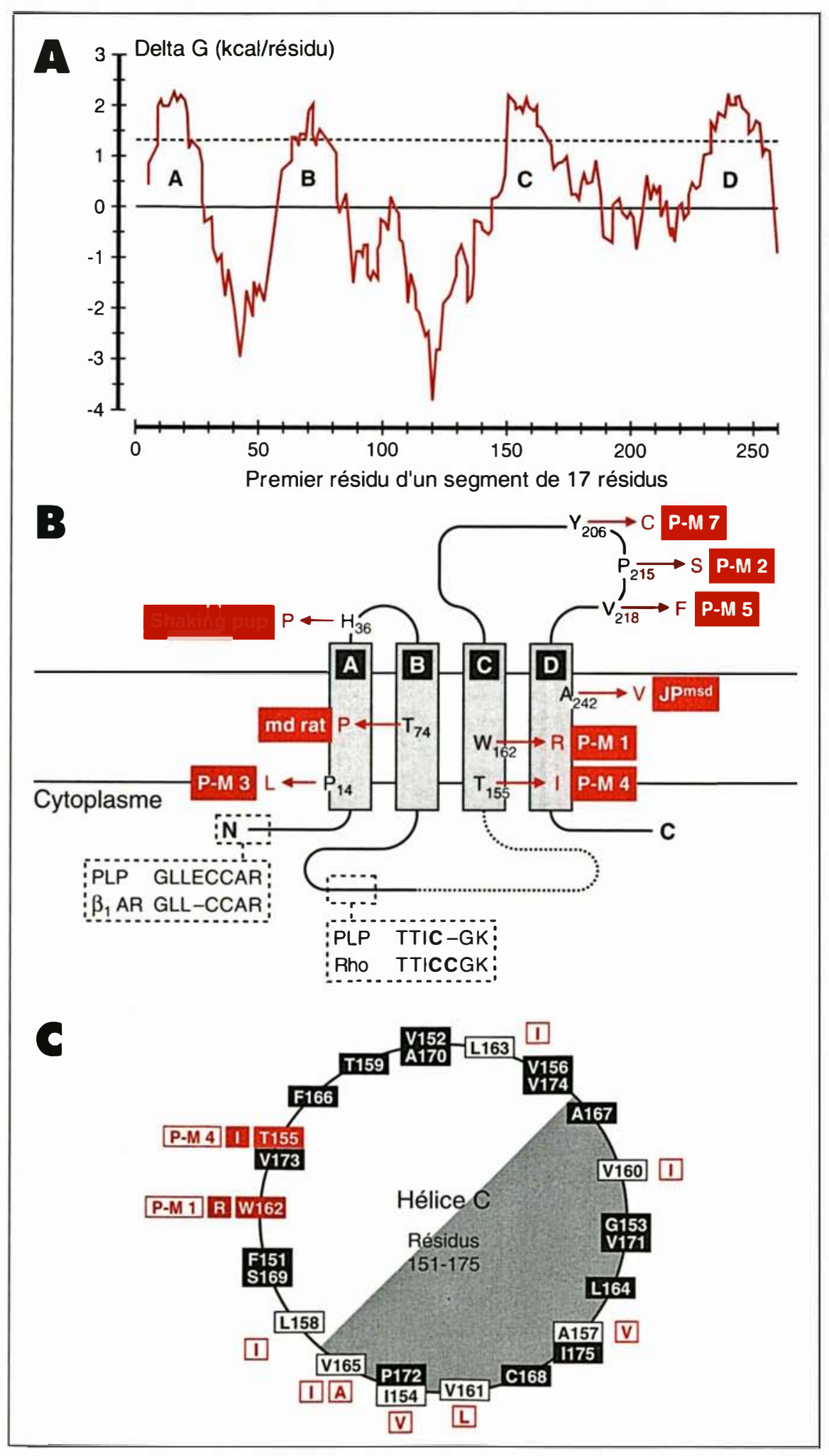

$\mathrm{m} / \mathrm{s} n^{\circ} 7$, vol. 8, septembre 92
En ce qui concernc le rôle de la DM20, on pcut fairc l'hypothèse que cettc isoforme joucrait un rôle autre qu'architectural à une période antéricure à la myélinisation, au cours du développement du système nervcux $[24,26]$. En effet, l'expression précoce de la DM20 par rapport au PLP [32] et le fait que toutes les mutations publiées à ce jour touchent des régions de la séquence communes aux deux isoformes (figure $3 B$ ), suggèrent que c'cst au niveau de la DM20 que se situe le défaut primaire du phénotype mutant. La présence du messager DM20 a été décrite pendant la période embryonnaire chez la souris [33, 34], à un moment où les oligodendrocytes nc sont pas encore présents dans le SNC. Il cst connu que les neurones et les astrocytes synthétisent des facteurs trophiques qui influent sur la prolifération et la maturation des oligodendrocytes [35]. S'il était démontré que la DM20 est cxprimće dans un autre type cellulairc, avant l'apparition des oligodendrocytes, on pourrait faire l'hypothèse que sa mutation empêche, par une perturbation du métabolisme cellulairc, la production de ces facteurs solubles, entraînant ainsi ultérieurcment la mort des oligodendrocytes. Cette hypothèse est à rapprocher des cxpériences qui suggèrent que les progéniteurs d'oligodendrocytes jimpy peuvent récupérer en partie un phénotype normal, quand ils sont placés dans un environnement normal, que ce soit in vivo [36] ou in vitro [13].

Par ailleurs, on peut imaginer que la DM20 joue un rôle dans la réception ou la transmission d'un signal, provenant d'un autre type cellulaire, impliqué dans la différenciation des précurseurs des oligodendrocytes. Dans cet ordre d'idée, on pcut remarquer que, sclon le modèle à quatre hélices que nous avons proposé [4], la DM20 contient, comme le PLP, deux segments de séquence cytosoliques semblables à une région également cytosolique de certains réceptcurs, impliquée dans l'interaction avec des protéines $\mathrm{G}$ (figure $3 \mathrm{~A}$ et $[4,21])$. Chez les mutants, cette fonction putative de transfert de signal scrait compromise par l'absence de transport de la protéine jusqu'à la membrane plasmique de l'oligoden- 
drocyte, son repliement incorrect et/ou son instabilité.

\section{Conclusions}

Les découvertes récentes concernant le gène $P L P$ en font un modèle d'économie génétique dans lequel un même gène code pour une isoforme ayant une fonction structurale, et une isof orme intervenant peut-être dans la régulation de la mort et de la survie cellulaire. Comme on aura pu le constater, on ne comprend pas, à ce jour, la cause primaire de la mort des oligodendrocytes chez les patients atteints de Pelizaeus-Merzbacher et dans les modèles animaux. Toutefois, les progrès importants réalisés ces dernières années, notamment grâce aux méthodes de la génétique moléculaire, ont ouvert de nombreuses voies de recherche nouvelles. On devrait voir se développer, au cours des années à venir, l'examen du rôle différentiel de la DM20 et du PLP selon le type cellulaire et l'étape du développement, ainsi que l'étude des mécanismes aboutissant à la mort des oligodendrocytes, que ce soit par un effet toxique direct du PLP muté dans ces cellules ou par l'effet indirect de la privation de facteurs trophiques, ou encore par la perte d'une fonction au niveau du transfert de signal dans l'oligodendrocyte. Le recensement des variations interspécifiques du PLP et la cartographie des mutations provoquant les dysmyélinisations liées à l'X devraient fournir des indications sur l'importance fonctionnelle des différentes régions de sa séquence. L'étude expérimentale de l'organisation transmembranaire du PLP devrait permettre d'infirmer ou de confirmer la topologie proposée. Ces informations, assorties d'une modélisation structurale plus élaborée, devraient conduire à des hypothèses plus précises quant au rôle délétère des différentes mutations du gène PLP

Remerciements

André Dautigny remercie le Cnrs, l'Inserm, l'AFM et l'Arsep ; Jean-Luc Popet, le MRE ; Odile BoespflugTanguy, l'AFM, l'Inserm et la MGEN, pour leur soutien financier.

\section{Summary}

Mutations in the gene encoding myelin proteolipid proteins (PLP and DM20) and X-linked inherited dysmyelinating diseases

Mutations affecting myelination may allow some insights into the complexity of the biological processes involved. Molecular characterization, in mice, of two mutants that affect myelin formation in the central nervous system, jimpy and shiverer, has led to numerous studies on the structure, topology and functions of major myelin proteins. The predominant proteins in myelin are the myelin proteolipids (PLP and isoform DM20) and the myelin basic proteins (MBP), which together account for $70-80 \%$ of total myelin proteins. The jimpy mutation, as other Xlinked dysmyelinating diseases in animal and man (PelizaeusMerzbacher disease), is associated with a single-base substitution in the PLP gene. The most striking feature of these mutations is the death of oligodendrocyte cells, which suggests that the two isoforms encoded by the PLP gene may sustain two functions in the developing nervous system, first in the differentiation of glial cells, then in myelination. 